Bangladesh J. Plant Taxon. 28(2): 311-315, 2021 (December)

(C) 2021 Bangladesh Association of Plant Taxonomists

\title{
NEW RECORDS OF SOME EUGLENOID ALGAE FROM BANGLADESH
}

\author{
Md. Almujaddade Alfasane*, Ashika Akhtar, Maliha Mehnaz, \\ Mst. Ayesha, Z.N. Tahmida Begum and Mahmoud Moustafa, ${ }^{1,2}$ \\ Department of Botany, University of Dhaka, Dhaka-1000, Bangladesh
}

Key words: Euglenoid algae; Euglena; Phacus; Protaspis; Strombomonas; Trachelomonas; New records; Bangladesh.

\begin{abstract}
The following taxa belong to eight species under five genera of euglenoid algae, namely, Euglena anabaena Mainx., Phacus glaber Pochmann (Defl.) Posch, Phacus indicus Skvortzov, Protaspis obovata Skuja, Strombomonas lanceolata (Playfair) Deflandre, Trachelomonas hexangulata (Swir) Playf., T. obovata var. klebsiana Defl. and T. pseudocaudata Defl. are described here for the first time for Bangladesh.

\section{Introduction}

So far, A large number of euglenoid algae have been reported from the different habitats of Bangladesh (Alfasane and Khondker, 2007; Alfasane et al., 2010, 2021; Gani et al., 2012. Khondker and Alfasane, 2005; Islam and Alfasane 2002, 2003, 2004; Islam and Muniruzzaman, 1981). Recently a large number of collections of the euglenoid algae from different fresh water of habitats of Sylhet Division of Bangladesh have been made. The euglenoid species are described here, could not be recorded earlier from the areas selected or any other parts of Bangladesh. So these could be considered as new records for Bangladesh. The recorded species belonged to the genera Euglena, Phacus, Protaspis, Strombomonas and Trachelomonas. The samples were collected from different stations of Shari Goyain River, Piyain River and Madhabpur Lake of Sylhet Division of Bangladesh. The descriptions of the organisms are given below.

\section{Materials and Methods}

Samples for the present paper were collected from Shari Goyain River and Piyain River of Sylhet District and Madhabpur Lake of Moulvibazar District of Sylhet Division between May 2017 and April 2020. Samples were collected by sieving $100 \mathrm{~L}$ of sub-surface water through a plankton net having a mesh size $20 \mu \mathrm{m}$ and preserved with Lugol's solution. Organisms were photomicrographed with the help of a Nikon Optiphot, UFX-11A microscope with a Nikon FX35WA camera, Japan.
\end{abstract}

\section{Taxonomy}

\section{Class: Euglenophyceae; Order: Euglenales; Family: Euglenaceae; Genus: Euglena Ehrenberg}

\section{Euglena anabaena Mainx.}

(Dillard 2000, 17; Pl 6, Fig. 8, Pringsheim, 1956, 71, Fig. A)

Cell length 55-95 $\mu \mathrm{m}$, breadth $18-23 \mu \mathrm{m}$, bluntly spindle shaped to near cylindrical, anterior end rounded, each cell slightly narrowing at posterior end and attenuated. Posterior end tapering rather abruptly to a tail, pellicular striations fine, spirally disposed. Chloroplasts 8-14 irregularly lobed discs with pyrenoid, pyrenoid body sometimes elongated.

It is a new record for Bangladesh. Collection no. P-3(1), 05.05.2017, Piyain River.

"Corresponding author, E-mail: mujaddade@yahoo.com

${ }^{1}$ Department of Biology, College of Science, King Khalid University, 9004, Abha, Kingdom of Saudi Arabia

${ }^{2}$ Department of Botany, Faculty of Science, South Valley University, Qena, Egypt 


\section{Class: Euglenophyceae; Order: Euglenales; Family: Euglenaceae; Genus: Phacus Dujardin}

2. Phacus glaber (Defl.) Poch.

(Fig. 3)

(Huber-Pestalozzi 1955, 238, Pl. 55, Fig. 340; Dillard 2000, 57, Pl. 8, Fig. 10; Philipose 1988, 542, Fig. 44)

Syn. Phacus hispidulus (Eich.) Lemm. fa. glabrus Defl.

Cells broadly oval in cross section and elliptic in lateral ribs, anterior end broadly rounded with a median papilla, posterior end abruptly narrowed into a long, awl-like caudus, pellicle with rows of fine punctae, much longer plexiform, end-sting and by the wart less periplast. Cell wall smooth; paramylum two lateral pads; chromatophores small, numerous and discoid; cell (including caudus) $26.5 \times 14.5 \mu \mathrm{m}$; caudus alone $7 \mu \mathrm{m}$.

It is a new record for Bangladesh. Collection no. M-4(2), 06.08.2017, Madhabpur Lake, S8(3), 10.11.2017, Shari Goyain River.

3. Phacus indicus Skvor.

(Fig. 4)

(Huber-Pestalozzi 1955,174, 199, Pl. 40, Fig. 248c; Wołowski and Walne 2007, 74, Fig. 255, 256) Syn. Phacus caudata var. undulata Skv., P. caudata var. volicensis Drez.

Cell length $27-43 \mu \mathrm{m}$, breadth 17-22 $\mu \mathrm{m}$, asymmetric, ovoid front slightly tapered at the center with 1-2 deep notches, each cell oval in outline with few deep incisions at the rim, caudus straight and sharp at the posterior end.

It is a new record for Bangladesh. Collection no. S-5(1), 05.05.2017, Shari Goyain River.

\section{Class: Euglenophyceae; Order: Desmomastigales; Family: Protaspidaceae; \\ Genus: Protaspis Skuja}

4. Protaspis obovata Skuja

(Fig. 2)

(Huber-Pestalozzi 1955, 544, Pl. 140, Fig. 1114c)

Cell length $26-40 \mu \mathrm{m}$, breadth $17-25 \mu \mathrm{m}$, reversed ovate to oblong pear shaped, dorsiventral, very little or no flattened more in the posterior part. Front rounded, towards the back cells are slightly narrowed.

The genus is a new record for Bangladesh. Collection no. M-3(1), 07.05.2017, Madhabpur Lake.

\section{Class: Euglenophyceae; Order: Euglenales; Family: Euglenaceae; Genus: Strombomonas Deflandre}

5. Strombomonas lanceolata (Play.) Defl.

(Fig. 5)

(Huber-Pestalozzi 1955, 380, Pl. 79, Fig. 820; Alves-da-Silva and Bridi, 2004)

Syn. Trachelomonas lanceolata Playfair, T. fluviatilis Swir, bei Deflan., T. dangeardi Skv., T. curta Skv. var pascheri (Drez.) Skv., T. fluviatilis var. pascheri Drez.

Cell length 23-31 $\mu \mathrm{m}$, breadth 12-14 $\mu \mathrm{m}$. Body oblong ellipsoidal, leading forward into a short, obliquely truncated and smooth-edged collar, ending behind with a short, strong, pointed caudal spine. Cell wall smooth, yellowish-green wall; discoid chloroplasts, pyrenoids present.

It is a new record for Bangladesh. Collection no. M-1(2), 06.08.2017, Madhabpur Lake. 

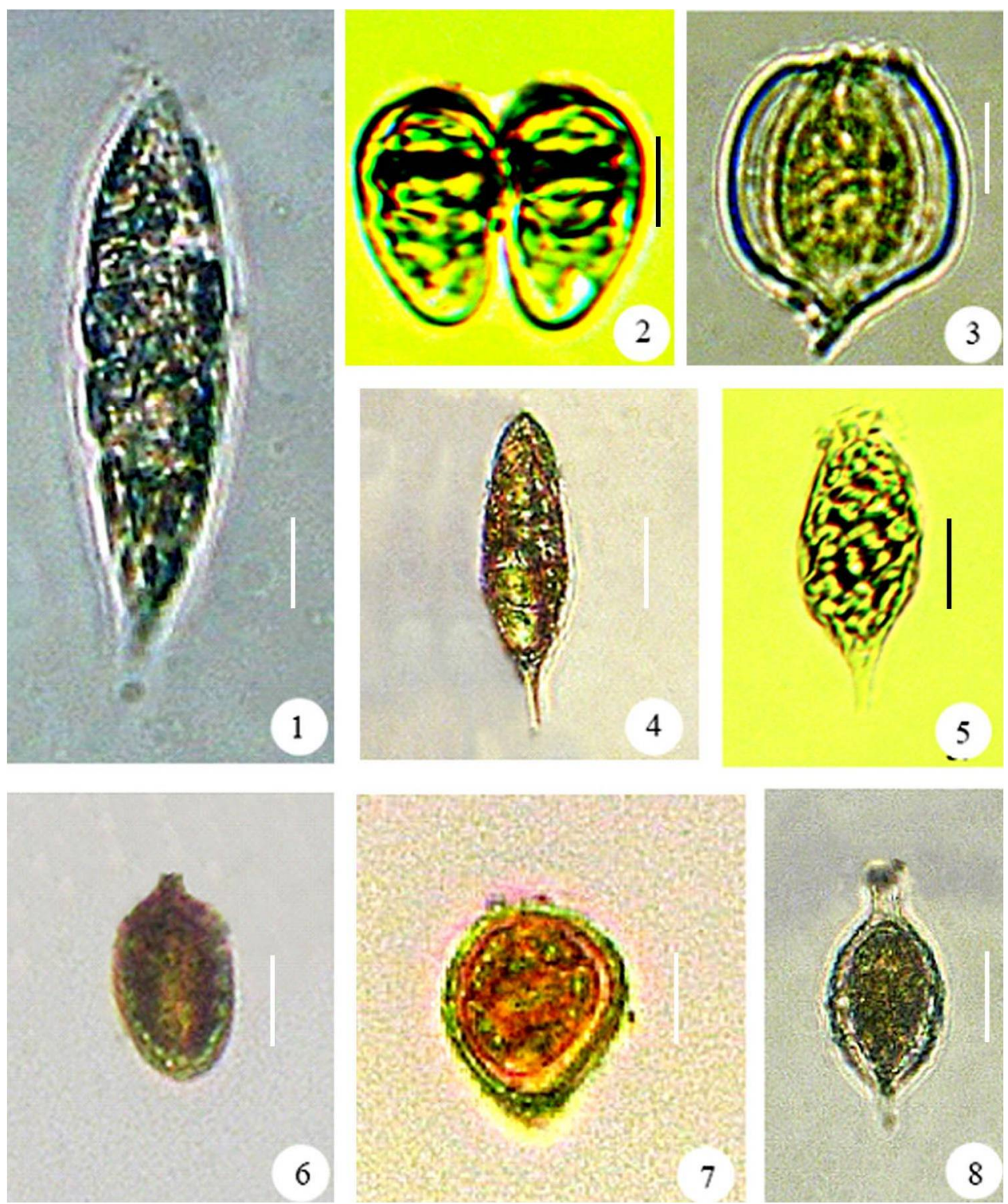

Figs 1-8. 1. Euglena anabaena Mainx., 2. Protaspis obovata Skuja, 3. Phacus glaber Pochmann (Defl.) Posch, 4. Phacus indicus Skvortzov, 5. Strombomonas lanceolata (Playfair) Deflandre, 6. Trachelomonas hexangulata (Swir) Playf. 7. T. obovata var. klebsiana Defl. 8. T. pseudocaudata Defl. (Scale=10 $\mu$ m).

Class: Euglenophyceae; Order: Euglenales; Family: Euglenaceae;

\section{Genus: Trachelomonas Ehrenberg}

6. Trachelomonas hexangulata (Swir) Playf.

(Fig. 6)

(Huber-Pestalozzi 1955, 335, Pl. 72, Fig. 702, Dillard, 2000, 82, Pl. 20, Fig. 1; Philipose 1988, 362, Pl. 10, Figs 2-6)

Syn. T. ampullula Playf. 
Cell length $24-30 \mu \mathrm{m}$, breadth $10-16 \mu \mathrm{m}$. Cell in frontal view approximately hexagonal, with rounded corners, sides in the middle part almost parallel. Cell collar height 2-5 $\mu \mathrm{m}$, front and hind ends conical; hind pole more or less truncate or sometimes broadly rounded; collar cylindrical, straight or sometimes with a membrane cone joining the collar wall with the upper margin of the lorica; membrane smooth and light yellowish.

It is a new record for Bangladesh. Collection no. P-7(4), 03.02.2018, Piyain River, S-7(2), 04.08.2017, Shari Goyain River.

7. T. obovata var. klebsiana Defl.

(Fig. 7)

(Huber-Pestalozzi 1955, 316, Pl. 69, Fig. 618; Dillard, 2000, 95, Pl. 15, Fig. 9)

Syn. $T$. reticulata Klebs fa. Defl..

Cell length 23-31 $\mu \mathrm{m}$, breadth 17-19 $\mu \mathrm{m}$, broadly or narrowly ovoid, without chromatophores, with or without paramylon, shell narrow or wide egg shaped.

It is a new record for Bangladesh. Collection no. S-2(3), 10.11.2017, Shari Goyain River.

8. T. pseudocaudata Defl.

(Fig. 8)

(Huber-Pestalozzi 1955, 351, Pl. 75, Fig. 755)

Cell length $40-42 \mu \mathrm{m}$, breadth $21-23 \mu \mathrm{m}$, collar 5-6 $\mu \mathrm{m}$ in height, breadth at the base 5-5.5 $\mu \mathrm{m}$, end extension 3.5-6.5 $\mu \mathrm{m}$. Shell regularly ellipsoidal, densely covered with stalk-shaped blunt spines. Pore surrounded by an annular thickening and a distinct cylindrical $(5-5.5 \mu \mathrm{m})$ collar which may be denticulated or not, posterior end with a short tail (3-4 $\mu \mathrm{m}$ long), sometimes truncated.

It is a new record for Bangladesh. Collection no. S-7(2), 05.02.2020, Shari Goyain River.

\section{Acknowledgements}

The authors thank the Deanship of Scientific Research at King Khalid University forfunding (R.G.P2/90/41).

\section{References}

Alfasane, M.A. and Khondker, M. 2007. New records of Phytoplankton for Bangladesh: Phacus, Lepocinclis and Pteromonas Bangladesh J. Plant Taxon. 14(2): 167-169.

Alfasane, M.A., Islam, M.S. and Khondker, M. 2010. Some freshwater phytoplankton as new reports from Bangladesh. Bangladesh J. Plant Taxon. 17(1): 87-92.

Alfasane, M.A., Mehnaz, M., Akhtar, A., Ayesha, M., Shafi, S.A., Islam, S., Begum, Z.N.T. and Moustafa, M. 2021. New records of Euglenophyceae for Bangladesh. Bangladesh J. Plant Taxon. 28(1): 11-15.

Alves-da-Silva, S. M. and Bridi, F. C. 2004. Euglenophyta no Parque Estadual Delta do Jacuí, Rio Grande do Sul, Sul do Brasil. 3. Gênero Strombomonas Defl.. Acta Botanica Brasilica. 18(3): 555-572.

Dillard, G. E. 2000. Freshwater algae of the Southeastern United States. Part 7. Pigmented Euglenophyceae. Bibl. Phycol.Bd. 106. J. Cramer, Berlin, Stuttgart, 135 pp. +20 pls.

Gani, M.A., Alfasane, M.A. and Khondker, M. 2012. New records of Euglenophyceae for Bangladesh. Bangladesh J. Plant Taxon. 19(1): 85-88.

Huber-Pestalozzi, G. H. 1955. Das Phytoplankton des Süsswassers. Euglenophyceen. Stuttgart (reprinted 1979) 16(4): 1-1135

Islam, A.K.M. Nurul and Alfasane, M.A. 2002. Euglenophyceae from Barisal district, Bangladesh: I. Genus Phacus. Bangladesh J. Plant Taxon. 9(2): 3-18.

Islam, A.K.M. Nurul and Alfasane, M.A. 2003. Euglenophyceae from Barisal district, Bangladesh: II. Lepocinclis, Strombomonas and Trachelomonas. Bangladesh J. Plant Taxon.10(1): 15-26. 
Islam, A.K.M. Nurul and Alfasane, M.A. 2004. Euglenophyceae from Barisal district, Bangladesh:III. Genus Trachelomonas Ehr. Bangladesh J. Plant Taxon.11(2): 33-37.

Islam, A.K.M. Nurul and Muniruzzaman, K. 1981. Euglenophyta of Bangladesh. I. Genus Trachelomonas Ehr. Int. Revue ges. Hydrobiol. 66(1): 109-125.

Khondker, M. and Alfasane, M.A. 2005. Euglenamorpha hegneri Wenrich (Euglenaceae): A rare euglenoid from Bangladesh. Bangladesh J. Bot. 34(1): 41-43.

Philipose, M.T. 1988. Contributions to our knowledge of Indian algae--3. Euglenineae Part 3. The genera Trachelomonas Ehrenberg and Strombomonas Deflandre. Proc. Indian Acad. Sci. Plant Sr. 98(5): 317 394.

Pringsheim, E.G. 1956. Contribution towards a monograph of the genus Euglena. Nova Acta. Leopol. Nov. Ser. 18: $3-168$.

Wołowski, K. and Walne, P.L. 2007. Strombomonas and Trachelomonas species (Euglenophyta) from south-eastern USA, Europ. J. Phycol. 42(4): 409-431.

(Manuscript received on 5 July 2020; revised on 4 July 202) 